\title{
A study of the minimum safe stopping distance between vehicles in terms of braking systems, weather and pavement conditions
}

\author{
Mansour Hadji Hosseinlou ${ }^{1}$; Hadi Ahadi ${ }^{2}$ and Vahid Hematian ${ }^{3}$ \\ Transportation Dept., Faculty of Civil Eng., K.N.Toosi University of Technology, Vali_Asr St., Mirdamad Cr., Tehran, Iran. \\ mansour@kntu.ac.ir ${ }^{1}$,hadiahadi@yahoo.com², vahid.hm@gmail.com ${ }^{3}$
}

\begin{abstract}
One of the most important factors in reducing traffic accidents is to keep safe distance between vehicles. The minimum safe distance depends on many factors such as the drivers' ability to react in accident, the vehicle braking system, the condition of the tires, the corresponding frictional forces provided by the tires, the speed of vehicles and many other factors. The main purpose of this study is to evaluate the above mentioned factors and obtain a relationship between speed and the so called minimum safe distance. In this study "Gim \& Nikravesh" and "Sakai" models have been used in order to calculate the braking system force in each tire, using twowheel equivalent model to calculate the braking force of vehicles and dynamic relations are used to calculate safe stopping time and distance between vehicles. Also the comparison of the minimum calculated safe distance with the relations delivered in Europe, Iran and British Codes are investigated. In this study, safety distance software has been introduced in order to calculate safe stopping distance based on different conditions. This software is designed and compiled by Delphi programming Environment and includes testing different hypothesizes for the back and front tires, calculating details of braking process such as stopping distance and stopping time, separating reflection distance and dynamic stopping distance, simulation of the stop process 3 seconds before confronting danger and finally drawing graphs related in the desired position. There is also a presentation of a recommendable formula in order to calculate the amount of safe distance for the two cases of ordinary braking system and "ABS"(Anti-lock Braking System) which covers the four situations of dry, wet, snowy, and icy positions.
\end{abstract}

Keywords: Simulation model, Vehicle motion, Tire model, Two wheel equivalent model, Safe stopping distance

\section{Introduction}

The ever increasing traffic volume of metropolitans in Iran, it is required to establish a regular and scientific methodology to mitigate the accident levels and to maximize the safety in urban traffic. Researchers have shown that the most of urban and rural accidents occur as a result of not observing safe distance between vehicles and lack of drivers' knowledge of the correct safe distance. AASHTO defined stopping sight distance as the length of roadway that is visible for driver and it is composed of two components, the distance traveled by the vehicle from the moment that driver sights an object to the instant the brake applied (Brake reaction distance) and the distance traveled from the beginning of braking application to stopping the vehicle (Braking distance) (A Policy on Geometric Design of Highway and Streets, 2004). The basic model based on the mentioned criteria was formulized in 1940 (A Policy on Sight Distance for Highways,1940) and the model parameters such as drivers eye height, object height and driver behavior have been altering since 1940. Many studies have questioned the validity of the model (Olson et al., 1984; Hall et al., 1988) and also tried to modify the model to represent the real-world situations. In 1997 Daniel B. Fambro et al. presented a new model for determining stopping sight distance based on the parameters describing driver and vehicle capabilities that can be validate with field data. Their model defined the stopping sight distance as a relationship between initial vehicle speed, driver perception-brake reaction time and vehicle deceleration (Fambro et al., 1997a). Later many studies have investigated the braking performance and driver performance during the application of braking on different pavement conditions (Fambro et al., 1997b; Koppa et al., 1996; Carlos E. Agudelo \& Eduardo Ferro, 2005; Zimmer and Ivey,1983; Quinn and Hilderbrand, 1973). Determining the stopping sight distance more accurate and based on the real-world situations is not only important for highway designers but also it is significant for police administrations and road users in terms of safety. Of course the lack of clear codes of traffic police administration documents contributes to the problem of determining the safe stopping distance. In this research, based on the Kwanghun Gim and Parviz E. Nikraveshtire model (KwanghunGim \& Parviz E. Nikravesh, 1991), Abe Masato (2009) motion model and Newton equations, the suggested model for the calculation of safe stopping distance is presented and the conclusions of this model will be compared with the calculated amounts based on Iranian and British Codes. 


\section{Effective factors in safe stopping distance}

Safe stopping distance encompasses of these two sections, driver reflection and dynamic distance of vehicle stop. Three factors are regarded as the main factors in final stopping distance and all of the other factors influence on safe stopping distance through these three factors. These three factors are as follow; human performance, vehicle and environmental conditions.

In determination of the distance of driver reflection the most important factor is the time of driver reflection; of course some factors are also important such as sense of seeing and hearing, watching accuracy soberness, age related sight error and other factors. Effective factors on vehicle performance are such as superficial factors including dimensions, weight, tire etc. and technical factors such as braking system, power, and gravity center. Also in case of environmental conditions some factors such as pavement conditions, wet and hot weather are important. Vehicle braking system includes ordinary braking system and ABS. In addition to preserving vehicles control in critical conditions by ABS, dynamic stopping distance will dramatically reduce (Broughton C. Baughan, 2002).

The important factor is that the "Speed" factor will be an irremovable factor in both sections of the distance of driver reflection and stopping dynamic distance and finally the last stopping distance will be calculated based on the presented equations based on speed.

In the section of stopping dynamic distance, European code (ECER13) has presented a table based on the kind of vehicle. Based on this table the equation ofdynamic distance calculation is the same as Eq. (1).

$$
D=0.1 V+V^{2} / 130
$$

Where $\mathrm{D}$ expresses stopping dynamic distance and $\mathrm{V}$ is the maximum available speed by vehicle $(\mathrm{km} / \mathrm{h})$ specified by manufacturer.

The goal of above equation is to preserve quality of produced vehicles by manufacturers via dynamic control of vehicle components. Observance of this criterion is obligatory for vehicles moving in streets of the European Union. According to purpose this paper for investigating the distance between vehicles in traffic flow and waiver performance of any vehicles, in this article solely plan and describing of this method have been mentioned regarding this object that Eq.(1) is completely simple and overestimated.

\section{Study method}

In this research based on Gim \& Nikraveshtire model, Sakai model (Takashi Nakatsuji et al., 2001), two-wheel equivalent motion model and Newton dynamic equations, computer simulation is implemented in Delphi programming environment and model results are derived in different graphs. Ultimately by way of investigating of equations presented in Iranian and British Code and compared them with model results, simple suggested models are presented by Mansour Farzam (2003) for measuring the minimum safe stopping distance.

\section{Model theory}

In order to calculate safe stopping distance the following calculations should be performed step by step.

- Calculation of testing brake force over each wheel (by the help of "Gim \& Nikravesh" and "Sakai" model).

- Calculation of resultant force testing over each vehicle (by the help of two wheel equivalent motion).

- Calculation of time and stopping distance (by the help of Newton dynamic equations).

The above models are regarded as the base of calculations in the designed software of "Safe Stopping Distance". The related descriptions for being familiar with this software will be mentioned in the next sections of this paper. In the calculations related in the above models which are aimed in the simplification of the supposed calculation, vehicles will not be diverted during the braking distance to the final stop and steering- wheel angle and rotation angle are zero, and vehicles will not rotate through the uniform function of each four wheel.

\subsection{Slip ratio relation with exerted force over tire}

Slip ratio(s) is the proportional motion between tire and road surface which relates to the way of braking. Slip ratio during braking will change from $0 \%$ (in situation when there has not been any braking) to $100 \%$ (in situation when the tire will be locked because of braking). In fact slip ratio shows the braking method of the driver.

Through braking function, slip ratio increases from $0 \%$ until 100\% (100\% shows locking situation of wheels). In practical view something which is effective in the amount of braking distance is opposite to motion force which we will take it from "Sakai" 
and "Gim \& Nikravesh" model in such a way that more force will lead to more negative speed of vehicle and the reduction of braking distance.

As it is shown in the Fig.1, 20\% slip ratio produces the most force in braking and this shows braking optimal position. This case of braking from the complete braking will have better performance with $100 \%$ slip ratio (tire locking) and it will reduce braking distance.Nowadays this idea is used in ABS and the best braking result will be produced for vehicle through keeping braking slip ratio around $20 \%$.

Fig.1. Proportion of tire force to tire weight force based on slip ratio.

Fig.2. Changes in safe stopping distance in 4 different pavement situations using anti-lock braking system.

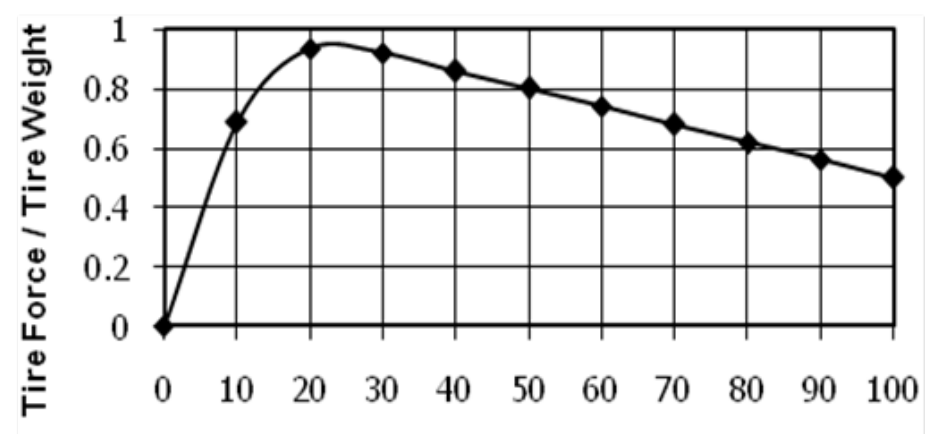

Slip Ratio [\%]

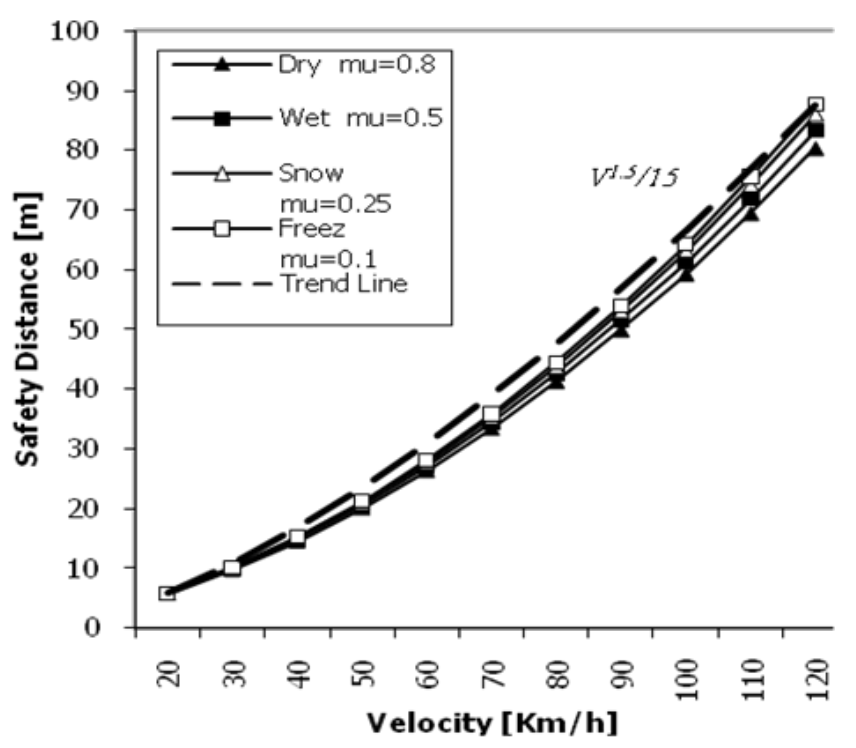

\section{Computer simulations}

In order to better understand vehicle stopping process better, software has been designed and presented by Delphi programming language. This software encompasses the following capabilities: 1) Variety of input data, the software also has the acceptance capability for different data in relation with back and front wheels. 2) Capability of automatic information entrance for Iranian conventional vehicles. 3) Representation of calculation separated results for the two models. 4) Stop simulation based on the two models. 5) Presentation of different graphs based on the two models. 6) Transforming the presented graphs in image files. And the possibility of printing those presented graphs.

Investigation of results acquired from computer calculation and based on mentioned models were studied separately for ordinary braking system and ABS braking system. Fig.2 shows ABS in four curves related in four different pavement conditions. These curves show the maximum amount resulted from "Nikravesh" and "Sakai" tire models.

Regarding very close relation of the results of two models in the two cases of lock and anti-lock. One curve resulted from more amount of this model is replaced with following curve related in two models.

So through drawing a covering curve titled in "Trend Duration" it is possible to present a fixed equation for all of the atmospheric conditions. The equation 2 which is presented below is the curve equation that is illustrated in Fig.2.

Where $\mathrm{D}$ expresses stopping dynamic distance and $\mathrm{V}$ is the maximum available speed by vehicle $(\mathrm{km} / \mathrm{h})$.

\section{$D=V^{\mathbf{L}} / 15$}

The only error of the above equation is that the drivers cannot rapidly calculate them during driving. Although it is possible to present a safe stopping distance table based on this equation similar to the British Code.

In ordinary braking system, situations are different. Fig. 3 shows the amount of safe stopping distance in four different pavement conditions at various velocities. The very great difference of safe stopping distance amount from $95 \mathrm{~m}$ in a dry condition to $580 \mathrm{~m}$ in an icy condition in speed of $120 \mathrm{~km} / \mathrm{h}$ is an obstacle for drawing an acceptable one covering curve and presenting unit equation for four conditions. In these conditions such as the previous case the appropriate equations with the supposed conditions are elicited through drawing safe stopping distance covering curve (Fig.4,5,6). The relations are presented in Table1. Based on the obligation of practicality of relations, it has been tried to have relations with simple appearance in order to have the capability of them remembering. 


\section{Comparison of model results based on Codes and Discussion}

\section{European Code}

European code (ECER13) has presented some equations for the calculation of vehicle dynamic stop distance. All of these equations are overestimated and encompasses the above models results.

\section{Iranian Code}

The existing equation in Iranian Code (2004) for safe stopping distance calculation between two moving vehicles is known as two seconds equation. Every driver based on this equation should keep his distance from the front vehicle as much as two second motions. So the amount of safe stopping distance will be calculated from Eq. (3):

$$
d=(V / 3.6) \times 2=V / 1.8
$$

Where D expresses safe stopping distance $(\mathrm{m})$ and $\mathrm{V}$ is the vehicle speed $(\mathrm{km} / \mathrm{h})$

Vehicle stop in any condition happen maximum in two seconds.

\section{British Code}

In The Highway code (2004) vehicle safe distance has been determined based on table2.

\section{Comparison of Iranian and British Codes with model results}

In order to compare model results by above Codes amounts with four different atmospheric conditions of dry, wet, snowy and icy are presented. Also based on safe stopping distance, high difference in using or not using ABS braking system, the two above conditions are separately investigated. In this research it supposed that the amount of friction coefficient between tire and road pavement is $0.8,0.5,0.25$ and 0.1 respectively in dry, wet, snowy and icy conditions. These comparisons are shown in Figs. 7 to 10 . A great safe distance of difference exists between the model and codes presented amounts specially in icy conditions when the reduction of friction coefficient will happen. The related results with ABS system does not have a great difference with code amounts in dry or in icy conditions. So it means that all of the presented equations in these codes have been based on this hypothesis that vehicles use ABS when still most of Iranian vehicles use old systems.

Fig.3. Relationship between velocity \& safe stopping distance in dry pavements using ordinary braking system.

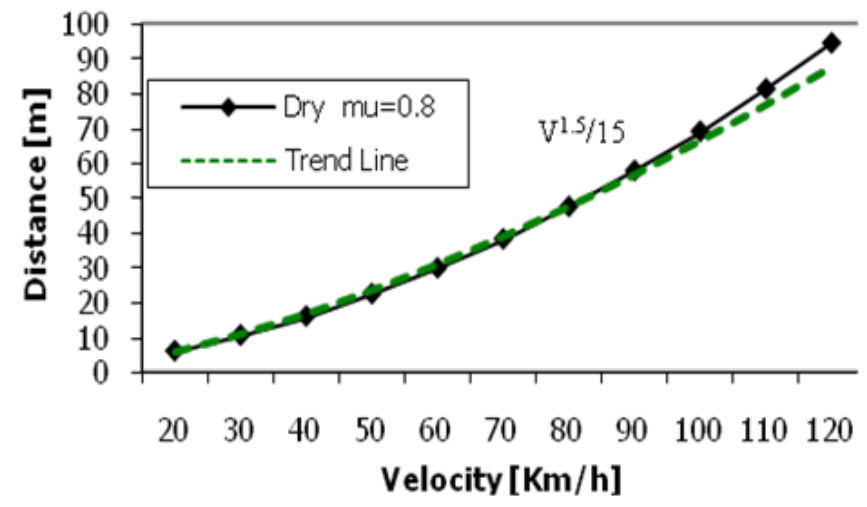

Fig.5. Relationship between velocity \& safe stopping distance in a snowy condition using ordinary braking system.

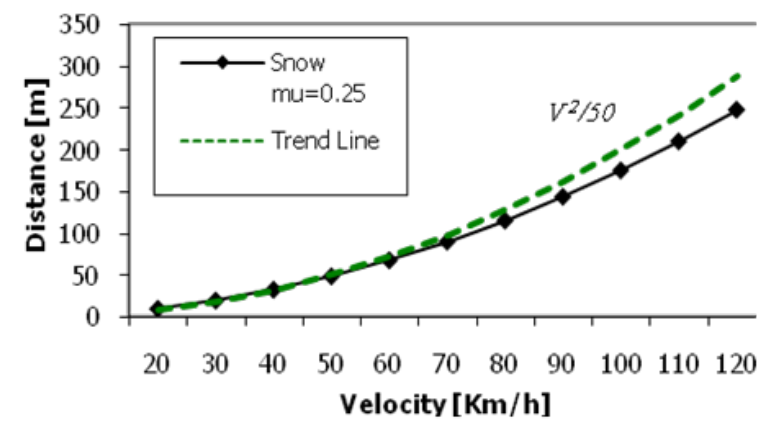

Fig.4. Relationship between velocity \& safe stopping distance in wet pavements using ordinary braking system.

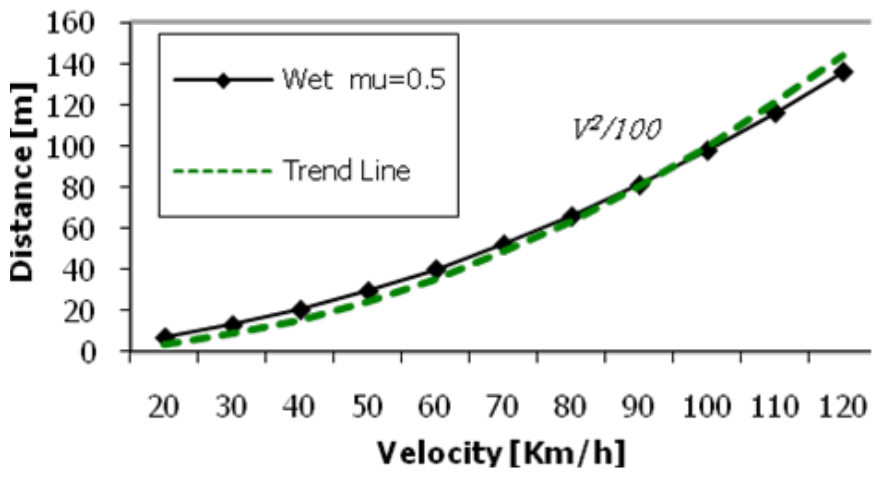

Fig.6. Relationship between velocity \& safe stopping distance in icy pavements using ordinary braking system.

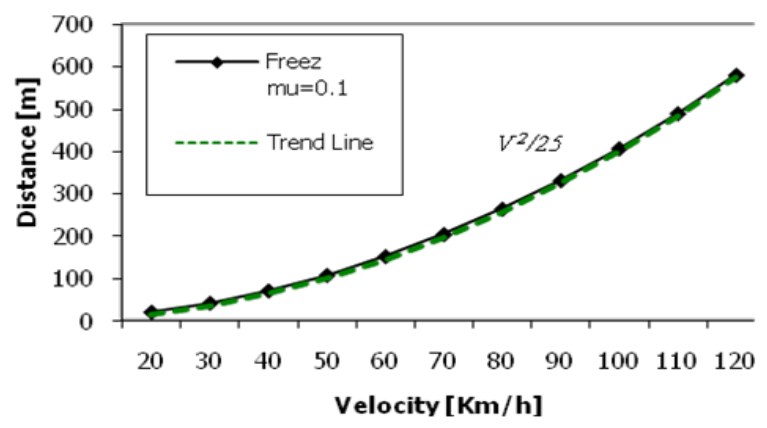


Table 1. Safe stopping distance calculation equations resulted from graphs

\begin{tabular}{|c|c|c|}
\hline Weather condition & anti-lock braking system & lock system (normal) \\
\hline \hline Dry & $D=V^{1.5} / 15$ & $D=V^{1.5} / 15$ \\
\hline Wet & & $D=V^{1.5} / 100$ \\
& & $D=V^{2 / 50}$ \\
& & $D=V^{2} / 25$ \\
\hline Snowy & \multicolumn{2}{|c}{} \\
\hline Icy & & \\
\hline \multicolumn{2}{|l}{ Note: D shows safe stopping distance (m) and V shows vehicle speed (km/h) } \\
\hline
\end{tabular}

Table 2. Safe distance codes in England

\begin{tabular}{|c|c|c|c|}
\hline $\begin{array}{c}\text { Velocity, } \\
\mathbf{k m} / \mathbf{h}\end{array}$ & Reaction distance, $\mathbf{m}$ & Stop distance, $\mathbf{m}$ & Safety distance, $\mathbf{m}$ \\
\hline 32 & 6 & 6 & 12 \\
\hline 48 & 9 & 14 & 23 \\
\hline 64 & 12 & 24 & 36 \\
\hline 80 & 15 & 38 & 53 \\
\hline 90 & 18 & 55 & 73 \\
\hline 113 & 21 & 75 & 96 \\
\hline
\end{tabular}

\section{Conclusion}

Given the road condition of icy and snowy, there is a great difference between the model calculated safe distance in ordinary braking system with those presented in British code and those resulted amounts from equation in Iranian code. However, if the ABS model of braking system is utilized, this difference is minimum. ABS performs in a way that there will not be great influence on safe distance in case of changes in atmospheric and pavement condition. In fact it can be said that since tire and road friction coefficient is not the main factor of stopping in ABS so increase or reduction in friction coefficient does not have influence on safe stopping distance. This means to eliminate the most of effective factors in the amount of minimum safe distance such as weather and moisture. Presentation of a fixed and permanent equation for the minimum safe stopping distance is correct and being advised just when braking system is anti-lock. Otherwise because of great difference of all pavement conditions, it is not possible to present a common equation for all of atmospheric conditions such as dry, wet, snowy and icy conditions. Using ABS will greatly reduce the required safe stopping distance. 
The amount of this reduction in icy conditions is more than six fold.

Fig.7. Comparison of model results bylranian and British Codes indry pavement.

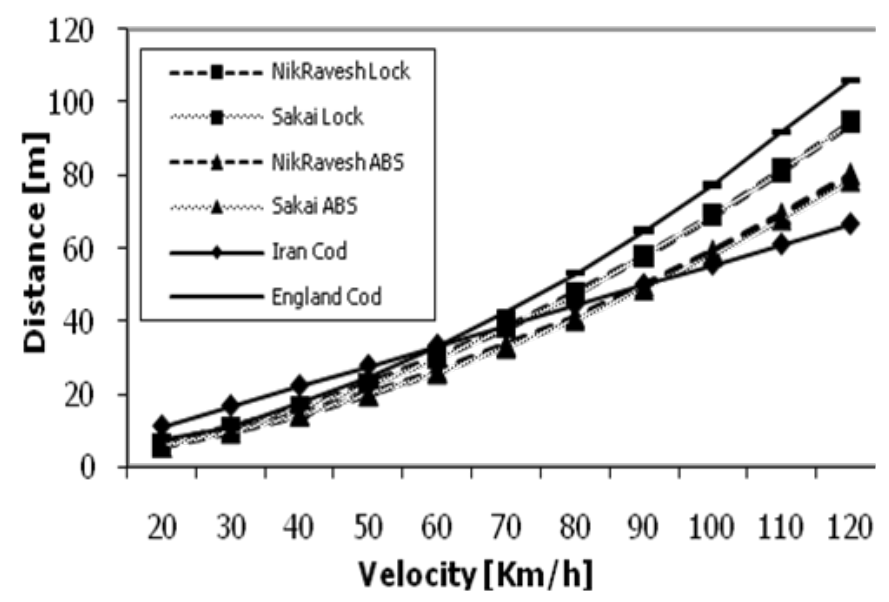

Fig.9. Comparison of model results by Iranian and British Codes in snowy condition.

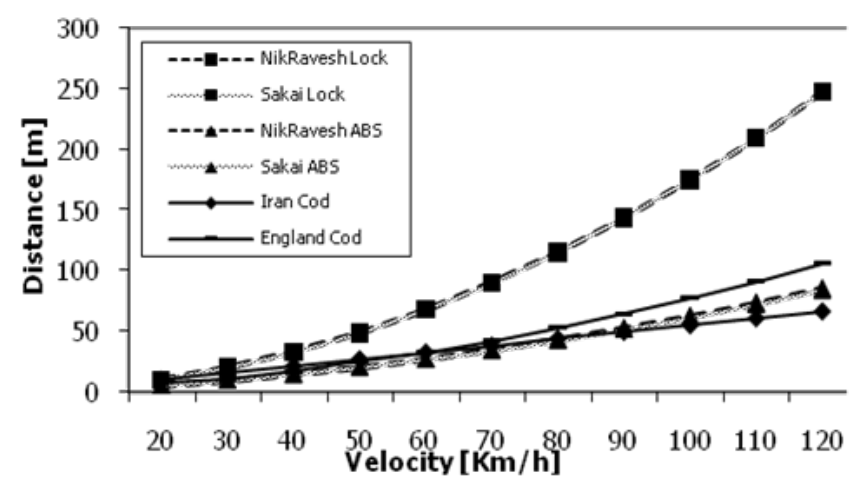

Fig.8. Comparison of model results by Iranian and British codes in wet pavement.

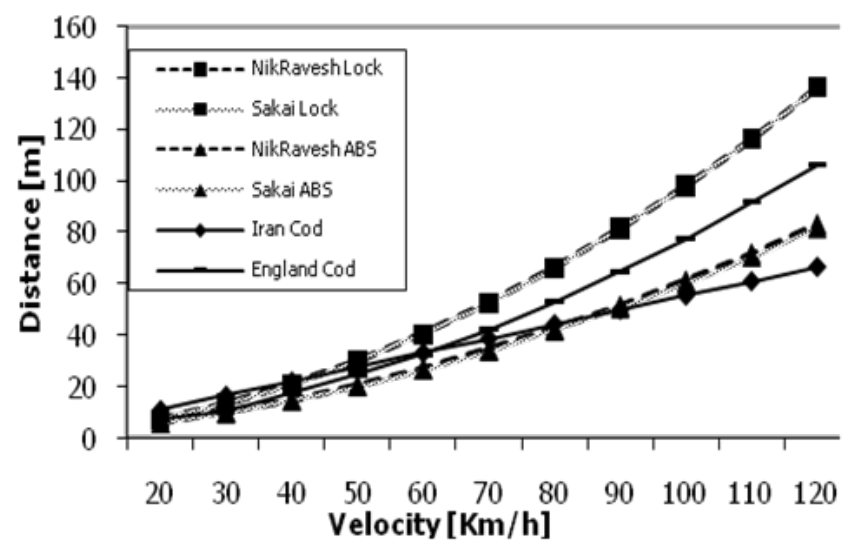

Fig.10.Comparison of model results by Iranian and British Codes in icy pavements.

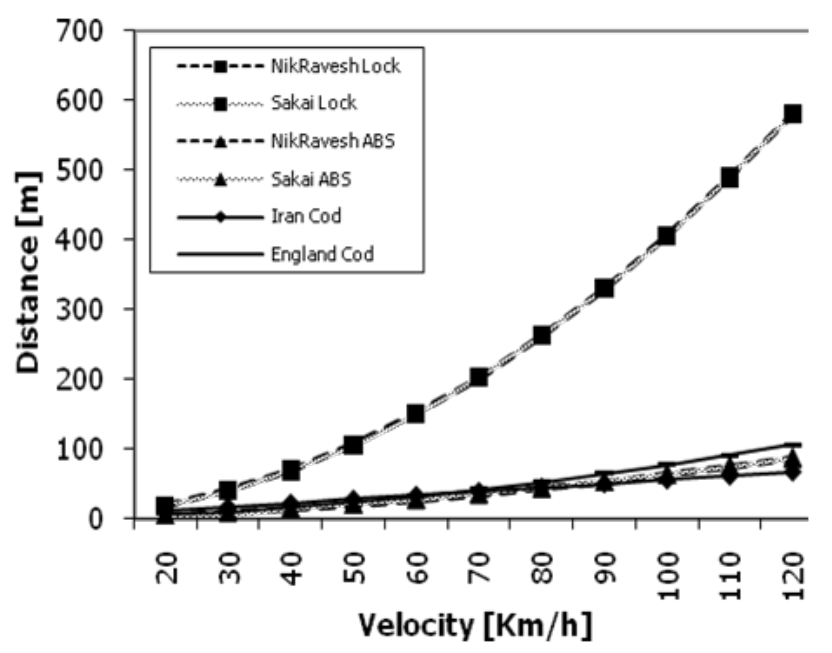

\section{References}

1 A Policy on Geometric Design of Highway and Streets (2004) American Association of State Highway and Transportation Officials, Washington, D.C.

2- A Policy on Sight Distance for Highways (1940) American Association of State Highway Officials, Washington, D.C.

3. Broughton J and Baughan C (2002) The effectiveness of antilock braking systems in reducing accidents in Great Britain, Accident analysis and prevention.

4 Carlos E. Agudelo and Eduardo Ferro (2005) Technical overview of brake performance testing for original equipment and aftermarket industries. In: The US and European Markets, Link Technical Report. pp: 1-27.

5- Fambro DB, Fitzpatrick K and Koppa R (1997a) A new stopping sight distance model for use in highway geometric design. Transportation Research Circular Transportation Research Board, National Research Council, Washington D.C.

6• Fambro DB, Koppa R, Picha D and Fitzpatrick K (1997b) Driver braking performance in stopping sight distance situations. Transportation Research Circular Transportation Research Board, National Research Council, Washington D.C.

7 Hall JW and Turner DS (1988) Stopping sight distance: Can we see where we now stand? In: Transportation Research Record 1208, TRB, National Research Council, Washington D.C. 4-13. 
8• Iran code (2004) Police publication.

9• Koppa RJ, Fambro DB and Zimmer RA (1996) Measuring driver performance in braking maneuvers. Transportation Research Record, Transportation Research Board, National Research Council, Washington D.C.

10 Kwanghun Gim and Parviz E. Nikravesh (1991) An analytical model of pneumatic tires for vehicles dynamics simulations. Int. J. Design. 12-1 vol? page?

11 Mansour Farzam (2003) How to study accidents. Police Publish.

12. Masato Abe (2009) Vehicle Handling dynamics.Butterworth-Heinemann.

13- Olson PL, Cleveland DE, Fancher PS, Kostyniuk LP and Schneider LW (1984) Parameters affecting stopping sight distance. In: NCHRP Parameters affecting stopping sight distance. NCHRP Report 270, Transportation Research Board, National Research Council, Washington, D.C.

14- Quinn BE and Hilderbrand SE (1973) Effect of road roughness on vehicle steering. Highway Res. Record. 47, 62-75.

15• Takashi Nakatsuji, Mansour HadjiHosseinlou, Akira Kawamura and Yuki Onodera (2001) Taking tire slip ratio into account and estimating friction coefficients. In: Rear-end collision on winter roads. J. the Transportation Res., Board 1741.

16• The Highway Code (2004) Stationery office books, 1st revised edition.

17 Zimmer RA and Ivey DL (1983) Pavement edges and vehicle stability: A basis for maintenance guidelines. Transportation Research Record 946, Report no, HS-037 874, 48-56. 